\title{
Detection of Freeze Injury in Oranges by Magnetic Resonance Imaging of Moving Samples
}

\author{
N. Hernández-Sánchez, P. Barreiro, M. Ruiz-Altisent, J. Ruiz-Cabello , \\ and M. Encarnación Fernández-Valle
}

\begin{abstract}
Physical Properties Laboratory, E.T.S.I.A., Polytechnic University of Madrid, Madrid, Spain Instituto de Estudios Biofuncionales. Universidad Complutense de Madrid, Madrid, Spain Centro de Asistencis a la Investigacłón de Resonancia Magnética Nuclear, Universidad Complutense de Madrid, Madrid, Spain
\end{abstract}

\begin{abstract}
Magnetic resonance imaging (MRI) is applied for on-line inspection of fruits. The aim of this work is to address the applicability of MRI for freeze injury detection in oranges directly on a distribution chain. Undamaged and damaged oranges are conveyed at 50 and $100 \mathrm{~mm} / \mathrm{s}$ by a specially designed conveyor within a $4.7 \mathrm{~T}$ spectrometer obtaining fast low-angle sho: images. An automatic segmentation algorithm is proposed that allows the discrimination between undamaged and damaged oranges.
\end{abstract}

\section{Introduction}

Fruit and vegetable quality assessment is becoming a mandatory condition in a worldwide market. The development of devices that are capable of evaluating different parameters for the definition of external and intemal quality is becoming a fundamental objective in this field $[1,2]$. Most available techniques used for internal quality measurement operate in a destructive way and have to be applied under an off-line strategy $(1 \mathrm{~min} / \mathrm{sample})$. Carrying out nondestructive measurements at low time cost would bring the product a desirable added value. Studies have shown that nuclear magnetic resonance (NMR) imaging allows a nondestructive study of the intemal properties of foods [3] including those of fruit and vegetables [4]. The possibility of developing on-line measurements as well as reaching working velocities that match those of the existing conveyors (below $1 \mathrm{~s} / \mathrm{sample}$ ) are indicated by several authors. NMR results are available for the real-time detection of pits in cherries [5], for the detection of bruises in apples [6] for the dynamic evaluation of the maturity stage in avocados [7] as well as for the detection of mealiness in apples [8]. 
This work rests on the importance of oranges cultivation with a production of 64 million tons worldwide, and the limitation of the growth of this subtropical fruit by the presence of low temperatures. Freezing injury of oranges may appear whenever a temperature below the freezing point of the tissues is reached during the preharvest growing period. Injured fruits usually remain on the tree without any visible external symptoms. For injured fruit the juice sacs dry out as the ice crystals burst the membranes and the cell walls. Also, the presence of water-soaking areas on the segment membranes becomes apparent for frozen fruits. At the severe stage, dried tissues collapse leading to the occurrence of hollows between and within the segments. The presence of both dehydration and hollows in damaged fruits indicates that magnetic resonance imaging (MRI) may be a suitable technique for freeze injury detection. This study follows works that have shown the viability of MRI to distinguish frozen-thawed products such as courgettes [9], blueberries [10] and kiwis [11] by means of changes in the contrast of the magnetic resonance images. In these studies the image acquisition was carried out from a static sample with no limitation in time. The objective of the present study is to develop a procedure that allows the identification of freeze injury in oranges in a moving conveyor in order to be applied for online sorting. For this reason there is a major need for the development of a sequence fast enough to minimize motion artifacts as well as to enable the discrimination between injured and healthy fruits. Additionally to this purpose, specific algorithms are developed for automatic characterization of the injury and for the establishment of the detection threshold.

\section{Material and Methods}

\section{I Samples}

Experimental work was developed on a set of 15 oranges potentially affected by freezing conditions during the growing season. Samples were picked from a local orchard. Another set of 6 unaffected oranges was obtained at a local market. Oranges were maintained at room temperature $\left(23^{\circ} \mathrm{C}\right)$ before measurements.

\subsection{MRI System and MRI-compatible Fruit Conveyor}

MRI experiments were performed on a Bruker Biospec 47/40 (Ettlingen, Germany) spectrometer operating at $200 \mathrm{MHz}(4.7 \mathrm{~T})$. This scanner is equipped with imaging gradients capable of reaching $30 \mathrm{mT} / \mathrm{m}$ along all axes. The bore of the magnet is horizontal with a $40 \mathrm{~cm}$ diameter reduced to $26 \mathrm{~cm}$ when the gradients stack in place. A birdcage coil with an inner diameter of $20 \mathrm{~cm}$ is used.

A special prototype conveyor that simulates the motion in an on-line inspection was designed and built to move the fruits through the spectrometer. The high magnetic field strength required the use of specific materials such as plas- 


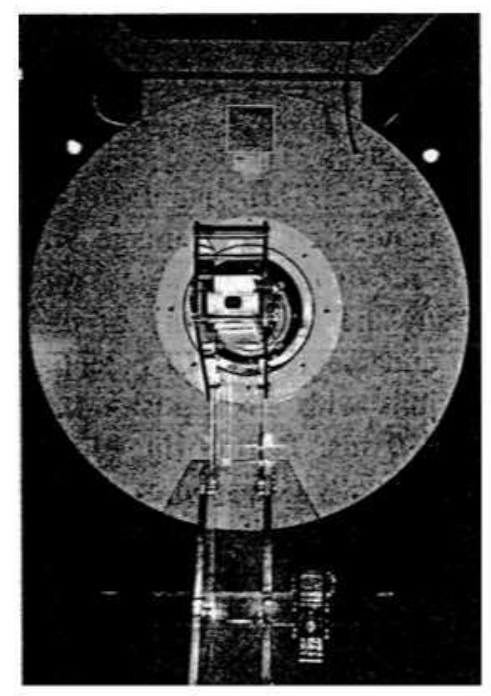

Fig. 1. Bruker Biospec $47 / 40$ and the custom-buik conveyor belt.

tic, nylon or bronze, exhaustively tested for high magnetic field applications in devoted trials, and all parts inside the MRI system (belt sensors, structure components, etc.) had to be nonmetal (Fig. 1). A stepper motor conducts the belt. An electronic device that allows a variable speed drive and bioptional direction of the motion, and the control of the signal triggering controls the motor. MRI signal acquisition is synchronized with fruit shifts by means of an electro-optic switch that delivers a digital signal to the controller.

\subsection{MRI Acquisition}

Samples were placed in the belt with their central axis (axis between the poles of the orange) along the $z$ direction. Pulse gains, attenuator and shimming settings were adjusted with the first sample and were held constant during the experiment. Oranges were imaged both under static condition (reference image) and during conveying through the magnet at different belt speeds.

The sample displacement inside the coil during the image acquisition generates a phase shift in the acquired $\mathrm{k}$-space lines that affects the outcome of the image reconstruction. This motion artifact is a frequent problem in MRI and is typically seen as ghosting and/or blurring of the image. Blurring due to motion may occur in any direction, causing the signal to be spatially dispersed [12]. A short acquisition time leads to a reduction in the displacement and therefore in the measured phase shift, with a consequent improvement in the image quality. With respect to such problems, a fast imaging sequence, i.e., fast low-angle shot (FLASH), would be helpful. 
The phase and the read encoding direction were selected along the $y$-axis and the $x$-axis, respectively, obtaining axial images at the equatorial area of the fruit zone. The recovery time TR was $12.2 \mathrm{~ms}$, the echo time TE was $3.8 \mathrm{~ms}$ and the pulse angle was 10 degrees. The field-of-view (FOV) was 12 by $12 \mathrm{~cm}$ and the slice thickness was $5.0 \mathrm{~mm}$. These parameters were the same under static as well as under dynamic acquisition. One image was obtained from each orange, setting the number of acquisitions for signal averaging to 1 ; three repetitions were performed to study the repeatability of the measurements.

The first images from 6 undamaged and 14 potentially damaged oranges were collected under static conditions with an acquisition matrix size 128 by 64 and a total acquisition time of $780 \mathrm{~ms}$. During the second set of experiments, the same 6 undamaged and 14 potentially damaged oranges were conveyed at $50 \mathrm{~mm} / \mathrm{s}$ and magnetic resonance images were collected with 128 by 64 acquisition points $(780 \mathrm{~ms})$. Finally, during a third set of experiments, images from 4 undamaged and 4 potentially damaged oranges were collected at 50 and $100 \mathrm{~mm} / \mathrm{s}$ as 128 by- $64(780 \mathrm{~ms})$ and 128 -by- 32 data arrays $(390 \mathrm{~ms})$. All of them were zerofilled to 128 by 128 points before reconstruction. In order to facilitate comparison between the different data sets, all images were reconstructed with an identical scale factor.

\subsection{Automated Image Analysis}

On-line inspection and calculation require an automated data analysis process that allows an unsupervised evaluation of the product. Thus, an algorithm was developed in order to extract several mathematical features for the characterization of each sample. The algorithm, developed using Matlab (The MathWorks, Inc.), exploits the effect of freezing conditions on the oranges, which generate dried flesh as well as internal hollows. The specific selection of pulse sequence and parameters makes these regions hypointense and reveals undamaged areas as having higher signal, highlighting a higher amount of juice. The algorithm should be capable of detecting the presence of damaged tissue and classify the severity of this injury. The algorithm is applied to the intensity signal images digitalized at 4 bytes.

The first step in the algorithm is to automatically select a region of interest (ROI) corresponding to the orange. The software removes the background pixels and the peel pixels, whose signal intensity is below an automated threshold. This threshold is computed under an iterative process as the averaged maximum signal corresponding to the pixels in the ten first and last columns of the matrix image multiplied by a variable factor. This variable factor increases through the iterative process, being smaller for each increasing time step. For each iteration the ROI is calculated. This area corresponds to the amount of pixels with signal values above the threshold. The end of the iterative process is achieved whenever the difference of the ROI areas for consecutives iterations is smaller than $2 \%$, that is, when stabilization in the computed area is reached (Fig. 2). The 


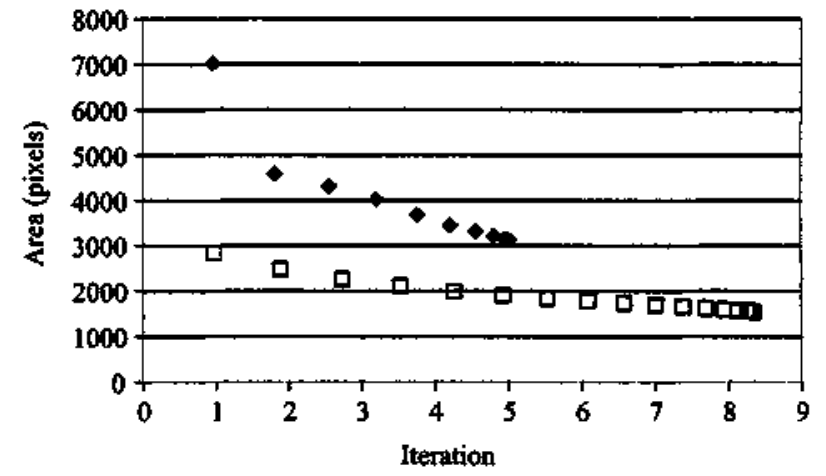

Fig. 2. Example of the relationship between computed areas in each iteration and the iteration step for both the ROI (diamonds) and the region corresponding to the undamaged tissue (squares).

selected ROI includes the initial holes in the segmented image. However, when extreme freeze damage is presented, the ROI does not include orange regions from the outer area of the flesh. For this reason a circular region of interest (CROI) is also computed within the automated algorithm. The center of gravity of the first ROI and its averaged diameter are calculated to generate the CROI (Fig. 3). This new region assures that most of the tissue is used for further calculations; although it may include several pixels from the peel, it allows a more reliable segmentation of the orange MRI data.

Once both regions of interest (ROI and CROI) have been obtained, the undamaged regions within these areas are identified. The sound tissue is easy to discem due to its internal homogeneity. The segmentation of such tissue within



Fig. 3. Example of the proposed segmentation in two areas. The grey area corresponds to the ROI and the external circumference (whose center is the center of gravity) delimitates the CROI. 
ROI and CROI is done by means of an iterative procedure similar to the one employed for the segmentation of ROI in the whole image. The procedure in this case uses only those pixels belonging to the ROI or CROI with larger iterative steps. The optimum threshold corresponding to ROI is low in order to select both damaged and undamaged tissues so that the final step factor value is smaller. In order to select sound tissue, the threshold is high so that the end of the iteration is reached at a higher step factor value. Afterwards, the damaged tissue is computed as the difference between ROI or CROI and the sound tissue. Two ratios are computed to evaluate the proportion of damaged tissue in the orange:

$$
\begin{gathered}
\frac{\mathrm{DT}}{\mathrm{ROI}}=\frac{\text { Damaged tissue (number of pixels) }}{\text { Region of interest (number of pixels) }}, \\
\frac{\mathrm{DT}}{\mathrm{CROI}}=\frac{\text { Damaged tissue (number of pixels) }}{\text { Circular region of interest (number of pixels) }} .
\end{gathered}
$$

Both ratios (DT/ROI and DT/CROI) are small for unaffected fruits and presumably of the same magnitude. This will allow one to establish a minimum threshold to characterize freeze injury in oranges. The thresholds are set on the basis of the measured standard deviation of these ratios for 3 repetitions. The selection can provide valuable clues for recognition of the level of damage severity. Three categories have been defined on the basis of the means of the pixel intensity histograms of the damaged areas in previously automatically selected images of injured oranges. The first one corresponds to the most severe damage (severity 1, S1); the second corresponds to the intermediate damage (severity 2, S2), and the third to the lightest damage (severity $3, \mathrm{~S} 3$ ). New ratios are computed as follows:

$$
\begin{aligned}
& \frac{\mathrm{S} 1}{\mathrm{ROI}}=\frac{\text { Severity } 1 \text { (number of pixels) }}{\text { Region of interest (number of pixels) }}, \\
& \frac{\mathrm{S} 1}{\mathrm{CROI}}=\frac{\text { Severity } 1 \text { (number of pixels) }}{\text { Circular region of interest (number of pixels) }}, \\
& \frac{\mathrm{S} 2}{\mathrm{ROI}}=\frac{\text { Severity } 2 \text { (number of pixels) }}{\text { Region of interest (number of pixels) }}, \\
& \frac{\mathrm{S} 2}{\mathrm{CROI}}=\frac{\text { Severity } 2 \text { (number of pixels) }}{\text { Circular region of interest (number of pixels) }}, \\
& \frac{\mathrm{S} 3}{\mathrm{ROI}}=\frac{\text { Severity } 3 \text { (number of pixels) }}{\text { Region of interest (number of pixels) }}, \\
& \frac{\mathrm{S} 3}{\mathrm{CROI}}=\frac{\text { Severity } 3 \text { (number of pixels) }}{\text { Circular region of interest (number of pixels) }} .
\end{aligned}
$$


Although the CROI fits better the surface of orange tissue, the ratios referenced to both ROI and CROI were calculated to allow comparison.

\subsection{Image Metrology Measurement}

The automatic identification procedure has to be consistent and accurate for reliable feature detection. Therefore, a robustness study is performed to analyze metrological parameters as the repeatability and the reproducibility indexes corresponding to the DT/ROI and DT/CROI measurements.

Repeatability is a measure of the ability of a sensor to reachieve the same response under similar experimental conditions. To study this parameter, three repetitions were taken at each belt speed, 50 and $100 \mathrm{~mm} / \mathrm{s}$, and for each acquisition matrix size, 128 by 64 and 128 by 32 . The average of the standard deviations of the computed ratios from each fruit was computed.

Reproducibility is a measure of the tendency of a sensor to provide the same response under changing experimental conditions (calibration, position, experimental day, etc.). This parameter was studied acquiring images (three repetitions) in two different days. The reproducibility error was calculated according to the STD of the averaged ratios between days. Furthermore, an analysis of variance (ANOVA) at the $5 \%$ significance level was applied to the computed ratios for these metrology experiments.

\subsection{Appointment of Reference Measurements}

After MRI, oranges were cut in half for visual inspection and reference classification using two measurements. The first value is the water volume syringed to fill internal voids as initial reference of the freeze injury severity. This value is associated with the total amount of hollows in an orange, while the damage identified through the segmentation of the MR images corresponds to a $5 \mathrm{~mm}$ stice in the equator of the fruit. The second value is the analysis of RGB images taken with a digital camera of the equatorial fruit section. This measurement was performed to compare the results from MRI in terms of freeze-induced dehydration, which occurs at an initial stage. RGB image analysis was applied to compute the dehydrated area represented by the green intensity component $\left(I_{g}\right)$ in the RGB color space. This component was analyzed as it offers the best contrast for dehydrated tissue when compared to the red or blue intensity components. The histogram segmentation of these images allows one to select this area and to establish the ratio to the whole tissue without peel, as in automated segmentation. The RGB intensity components were calculated as indicated below and used for further RGB segmentation:

$$
\begin{aligned}
& I_{\mathrm{r}}(x, y)=i_{\mathrm{r}}\left(i_{t}+i_{\mathrm{g}}+i_{\mathrm{b}}\right), \\
& I_{\mathrm{g}}(x, y)=i_{\mathrm{g}}\left(i_{\mathrm{r}}+i_{\mathrm{g}}+i_{\mathrm{b}}\right),
\end{aligned}
$$




$$
\left.I_{\mathrm{b}}(x, y)=i_{\mathrm{b}} / i_{\mathrm{r}}+i_{\mathrm{y}}+i_{\mathrm{b}}\right)
$$

where $i_{n}, i_{n}$ and $i_{\mathrm{b}}$ are the intensities obtained through red, green and blue filters, respectively.

Oranges show a high red component $I_{r}>0.55$ and a high green component $I_{g}>0.35$. RGB segmentation at a value of $I_{g}>0.37$ enhances the dehydrated tissue and allows a comparison with the results obrained by automated MRI segmentation.

\section{Results and Discussion}

As expected, the samples obtained from the market were undamaged. This was confirmed by the high and homogeneous signal intensity in MR images as well as by the inspection after cutting. Among the potentialty damaged oranges, only item number 1 and item number 10 showed small areas of very light decrease in signal intensity, while the rest showed a range from intermediate to large areas with low-intensity signal. Visual inspection on equivalent MRI slice revealed the presence of the dehydrated tissue and hollows corresponding to the hypointense signal intensity areas in these images. Table 1 shows the reference values measured in RGB images for freeze damage quantification.

Figure 4 shows MRI examples of both undamaged and damaged oranges. Figures 5 and 6 display the ratios DT/ROI and DT/CROI for several belt speeds and acquisition times. Ratios DT/ROI and DT/CROI show a similar sorting ten-

Table 1. Intertal hollow volumes and RGB segmentation.

\begin{tabular}{|c|c|c|c|c|c|}
\hline \multirow{2}{*}{ Sampie } & \multicolumn{2}{|c|}{ Undamaged orange } & \multirow{2}{*}{ Sample } & \multicolumn{2}{|c|}{ Potentially damaged orange } \\
\hline & Volume (ml) & $\mathrm{Dh} / \mathrm{ET}(\%)^{2}$ & & Volume (nt) & Dh/ET $(\%)^{\circ}$ \\
\hline Ut & 0.8 & 4.7 & P! & 1.45 & 5.1 \\
\hline U2 & 0.7 & 6.4 & $p 2$ & 2.5 & 17.1 \\
\hline U3 & 0.5 & 11 & P3 & 7 & 19.9 \\
\hline U4 & 1.1 & 4.7 & $p_{4}$ & 6 & 33 \\
\hline 45 & 1.2 & 2.6 & P5 & 3.1 & 17.5 \\
\hline \multirow[t]{10}{*}{ L6 } & 0.85 & 5.1 & P6 & 10 & 12.1 \\
\hline & & & P7 & 25 & 46.4 \\
\hline & & & P8 & 9 & 7 \\
\hline & & & pq & 25 & 15.4 \\
\hline & & & $\mathrm{P}[0$ & 3 & 4.8 \\
\hline & & & $P ! 1$ & 5 & 10.3 \\
\hline & & & $P ! 2$ & 8 & 6.3 \\
\hline & & & $\mathrm{Pis}$ & 25 & 14.1 \\
\hline & & & $\mathrm{PI} 4$ & 6.5 & 7.4 \\
\hline & & & PIS & 17 & 23.7 \\
\hline
\end{tabular}

- Dh, dehydrated tissue area: E.T, entire tissue area. 
Belt speed, $0 \mathrm{~mm} / \mathrm{s}$; acquisition time, $780 \mathrm{~ms}$
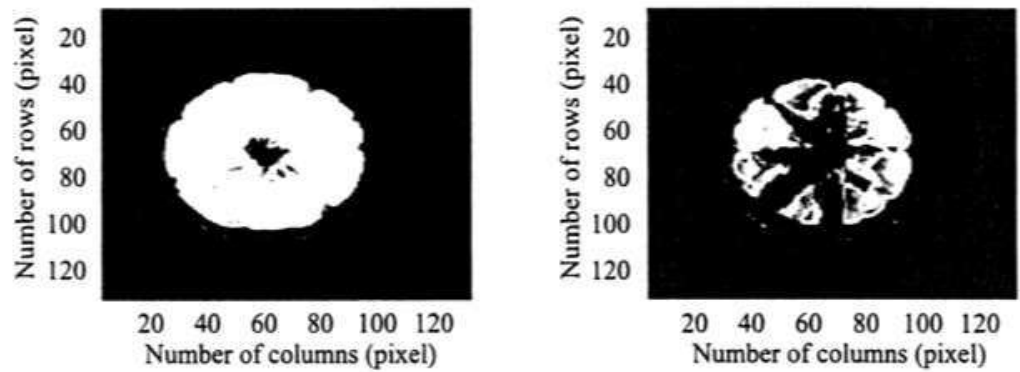

Belt speed, $50 \mathrm{~mm} / \mathrm{s}$; acquisition time, $780 \mathrm{~ms}$
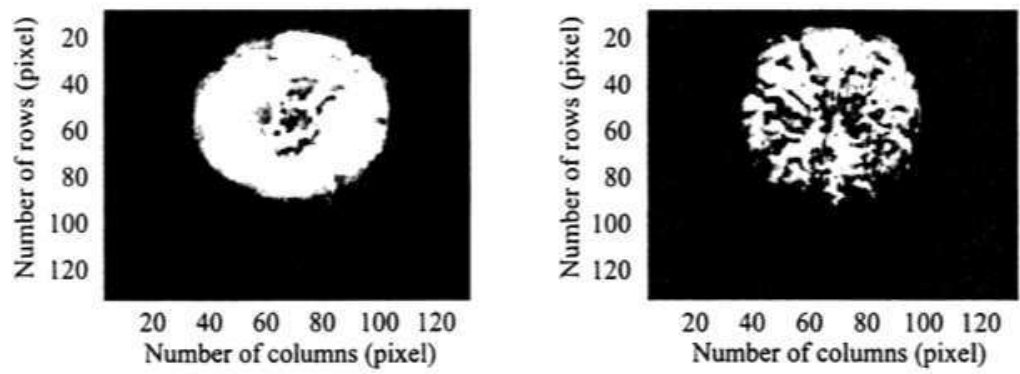

Belt speed, $100 \mathrm{~mm} / \mathrm{s}$; acquisition time, $780 \mathrm{~ms}$


Belt speed, $100 \mathrm{~mm} / \mathrm{s}$; acquisition time, $390 \mathrm{~ms}$


Fig. 4. MRI examples of both unaffected and affected oranges at different belt speeds and acquisition times. 

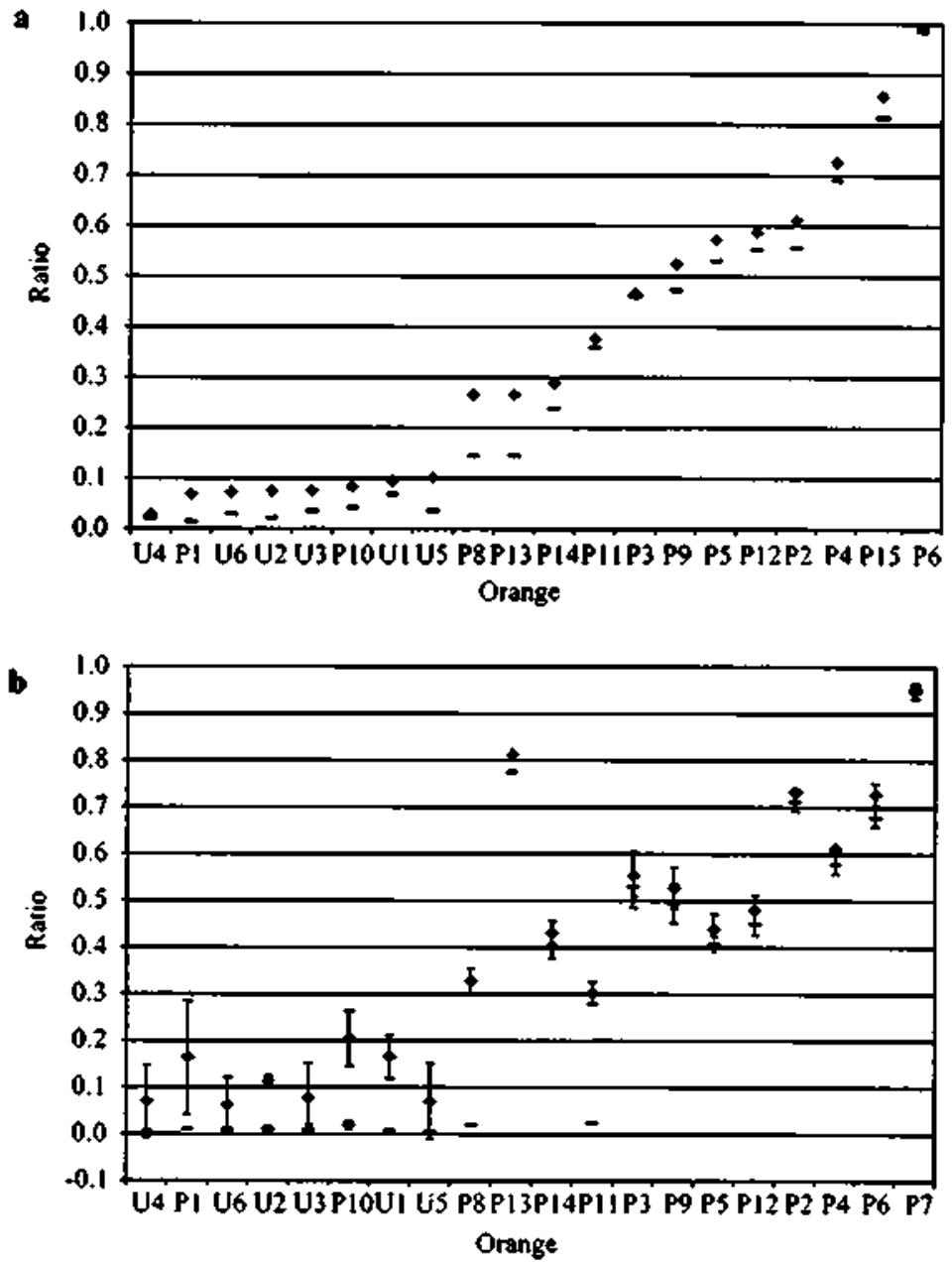

Fig. 5. Referenced ratios (DT/ROI. -: DT/CROL, $\bullet$ ) at different speeds belt and acquisition times. Vertical lines represent the standard deviation of three repetitions. a Belt speeds, $0 \mathrm{~mm} / \mathrm{s}$; acquisition times, $780 \mathrm{~ms}$. b Belt speeds. $50 \mathrm{~mm} / \mathrm{s}$; acquisition times, $780 \mathrm{~ms}$.

dency. However, the proportions of damaged area in relation to the ROl reach lower values than those calculated in relation to the CROI. This difference is due to the fact that in the first case damaged tissue in the outer zone of the flesh is not selected as part of the ROI.

In the nonaffected oranges, the percentage of damaged areas that appears in the automated analysis is related to the size of the central axis. In these cases the orange is classified as unaffected. No problems occured in the indentification of freeze-injured oranges when the area of the damage was above $8 \%$ of the ROI and $10 \%$ of the CROI under static condition. This value is established as a 

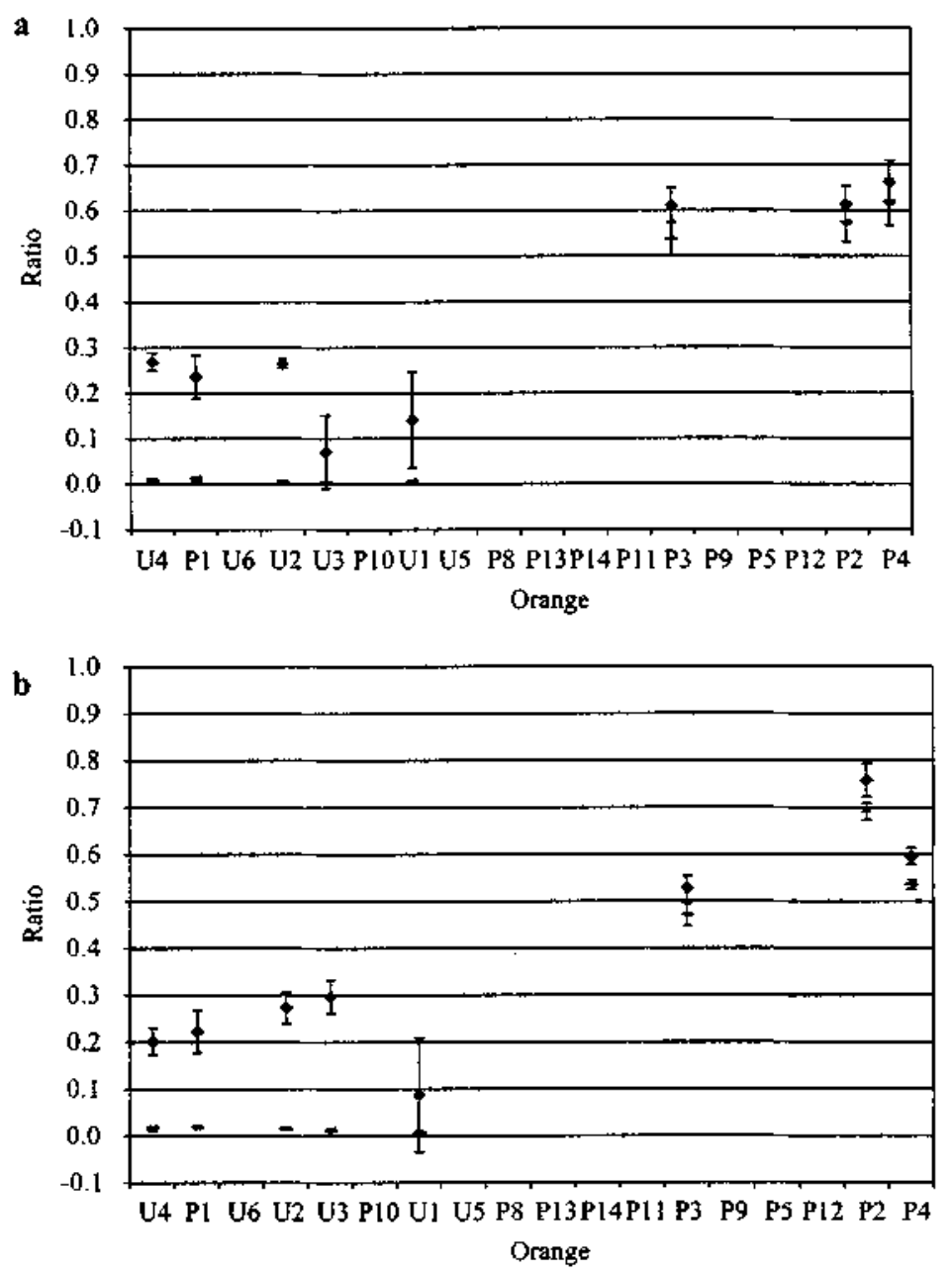

Fig. 6. Referenced ratios (DT/ROI, -; DT/CROI, $\bullet$ ) at different speeds belt and acquisition times. Vertical jines represent the standard deviation of three repetitions. a Belt speeds, $100 \mathrm{~mm} / \mathrm{s}$; acquisition times, $780 \mathrm{~ms}$. b Belt speeds, $100 \mathrm{~mm} / \mathrm{s}$; acquisition times, $390 \mathrm{~ms}$.

detection threshold for these conditions. Damaged tissue is divided into three groups of decreasing injury severity in relation to the averaged signal intensity of the sound tissue. The first group (S1) includes those pixels with signal intensity below $15 \%$, the second group (S2) refers to those pixels with signal intensity between $15 \%$ and $25 \%$, and the third group (S3) includes those pixels with a signal value above $25 \%$ of the averaged undamaged tissue, respectiveity. This classification allows us to sort the damaged area of the fruit into severity levels of damage and so to establish differences between oranges with the same injury extent. 


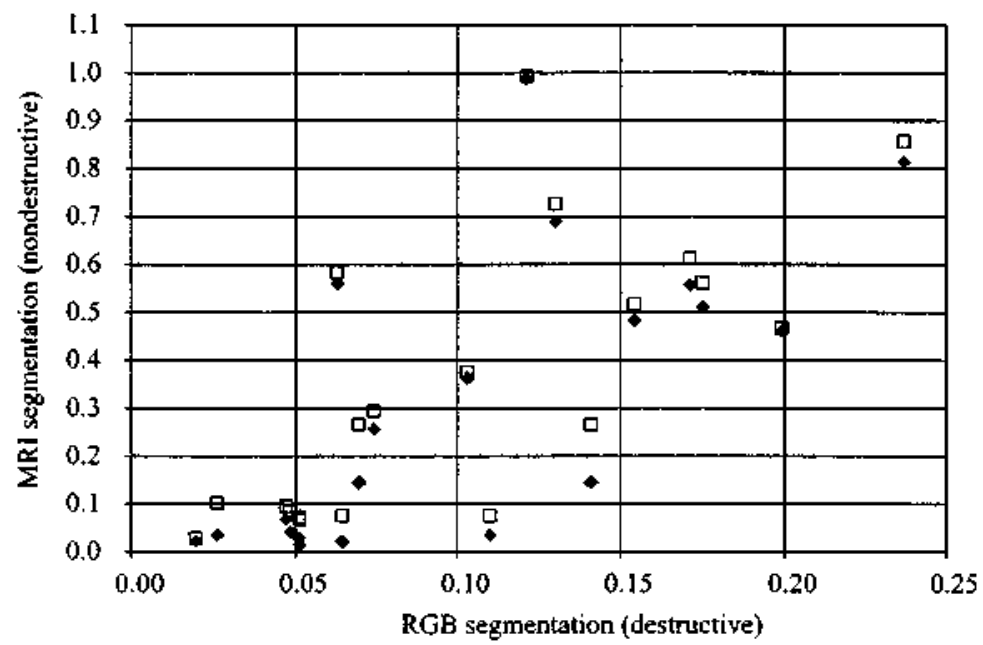

Fig. 7. Comparison of automated MR! segmentation (static conditions) and RGB segmentation. DT/ROI, ४; DT/CROI, ם.

The automatically computed results obtained from the MRI were linearly correlated (Fig. 7) to the declared reference parameters (volume of hollows and RGB segmentation). Table 2 shows that the volume of hollows and RGB seg. mentation have a high correlation coefficient $r$ with the ratios S1/ROI and S1/ CROI, i.e., with respect to the most damaged tissue. When $r$ is calculated for the MRI ratios of total damage, it is still high with RGB segmentation, while being poorly correlated with respect to the volume of hollows.

The first group of MR images obtained under dynamic conditions corresponds to a belt speed of $50 \mathrm{~mm} / \mathrm{s}$ and 128 by 64 acquisition data. FLASH images showed motion artifacts in the phase-encoding direction characterized by the superimposition of the signal arising from new tissue reaching the spatial position of the selected slice during the acquisition time. Despite this fact, differences in the signal can be appreciated by comparing damaged areas to sound areas, and segmentation was performed with reliable results. At this speed the DT/CROI ratio shows higher values than the DT/ROI ratio as expected.

Table 2. Linear correlation coefficient $r$ between reference measurements (hollow volune and RGB segmentation) under static conditions and MRI segmentation.

\begin{tabular}{ccccc}
\hline Reference & S1/ROI & S1/CROI & DT/ROI & DT/CROI \\
\hline Volume & 0.76 & 0.55 & 0.41 & 0.45 \\
Dh/ET & 0.71 & 0.71 & 0.70 & 0.72 \\
\hline
\end{tabular}

- Dh, dehydrated tissue area; ET, entire tissue area. 
Table 3. Repeatability error of the computed ratios.

\begin{tabular}{ccccc}
\hline Belt speed (mm/s) & Acquisition time (ms) & DT/ROI & DT/CROI & Nr. of fruits analyzed \\
\hline 50 & 780 & 0.0133 & 0.041 & 20 \\
100 & 780 & 0.0184 & 0.0491 & 8 \\
100 & 390 & 0.0095 & 0.0428 & 8 \\
\hline
\end{tabular}

The detection threshold can be established as $3 \%$ and $28 \%$ of damaged tissue in relation to the ROI and the CROI, respectively. For the "dynamic" MRI data, due to the severe artifacts of the images, no attempt to identify the damage severity level was performed.

A group of 4 undamaged and 4 potentially damaged oranges were also imaged at a belt speed of $100 \mathrm{~mm} / \mathrm{s}$ and a 128 by 64 acquisition matrix. The quality of the images decreased for the higher motion rate. The decrease in the quality of the images strongly affected the algorithm calculating the center of gravity and the ratios corresponding to the flesh of the orange and thus the ratios related to the CROI. The segmented ROI was not affected by either the high speed or the low image resolution and so were the ratios referenced to it. The threshold values are $2 \%$ and $30 \%$ of damaged area in relation to the ROI and CROI,

Table 4. Results of ANOVA ( $5 \%$ significance levei) for the reproducibility of measurements. The asterisk refers to the interaction between factors in the ANOVA analysis.

\begin{tabular}{|c|c|c|c|c|c|}
\hline Effect & $\begin{array}{l}\text { Surn of } \\
\text { squares }\end{array}$ & $\begin{array}{l}\text { Degrees of } \\
\text { freedom }\end{array}$ & Mean square & Fisher value & $\begin{array}{c}\text { Significance } \\
\text { level }\end{array}$ \\
\hline \multicolumn{6}{|c|}{ Reproducibility DT/ROI } \\
\hline latercept & 4.408837 & 1 & 4.408837 & 878.9637 & 0.000000 \\
\hline Class & 4.148332 & 1 & 4.148332 & 827.0284 & 0.000000 \\
\hline Repetition & 0.002487 & 2 & 0.001243 & 0.2479 & 0.781778 \\
\hline Test session day & 0.001118 & I & 0.001118 & 0.2229 & 0.639704 \\
\hline Class*Rep & 0.002629 & 2 & 0.001315 & 0.2621 & 0.770888 \\
\hline Class*Day & 0.000960 & 1 & 0.000960 & 0.1914 & 0.664328 \\
\hline Rep*Day & 0.001126 & 2 & 0.000563 & 0.1122 & $0.894 i 68$ \\
\hline Class*Rep*Day & 0.000710 & 2 & 0.000355 & 0.0707 & 0.931846 \\
\hline Etror & 0.180574 & 36 & 0.005016 & & \\
\hline \multicolumn{6}{|c|}{ Reproducibility DT/CROI } \\
\hline lntercept & 7.116059 & 1 & 7.116059 & 813.1478 & 0.000000 \\
\hline Class & 2.742067 & 1 & 2.742067 & 313.3344 & 0.000000 \\
\hline Repetition & 0.015006 & 2 & 0.007503 & 0.8574 & 0.432759 \\
\hline Test session day & 0.023434 & I & 0.023434 & 2.6778 & 0.110472 \\
\hline Class*Rep & 0.001768 & 2 & 0.000884 & 0.1010 & 0.904190 \\
\hline Class*Day & 0.004040 & l & 0.004040 & 0.4616 & 0.501201 \\
\hline Rep*Day & 0.007999 & 2 & 0.003999 & 0.4570 & 0.636793 \\
\hline Class*Rep*Day & 0.008965 & 2 & 0.004483 & 0.5122 & 0.603465 \\
\hline Error & 0.315045 & 36 & 0.008751 & & \\
\hline
\end{tabular}


respectively. These values agree with the problems stated for the computation of the CROI. In an attempt to reduce the motion effects, these oranges were imaged with a 128 by 32 acquisition matrix. As expected, the decrease in the amount of lines of the k-space generated a clear loss of image quality. However, as in previous acquisitions, a detection threshold to discriminate between unaffected and affected oranges was feasibly established. At this belt speed and data array the threshold is set to $3 \%$ and $35 \%$ of damaged area compared to the $\mathrm{ROI}$ and CROI, respectively. Note that again the CROI is highly affected by the image quality and so the threshold for damage detection increases.

To study the robustness of the technique for freeze damage detection, the repeatability and the reproducibility errors of the ratios DT/ROI and DT/CROI were computed (see Table 3 ). The repeatability error is around $1 \%$ of damaged tissue related to the ROI and $4 \%$ related to the CROI at $50 \mathrm{~mm} / \mathrm{s}$ and matrix size of 128 by 64 . The repeatability error increases to $2 \%$ for DT/ROI and $5 \%$ for DT/CROI at $100 \mathrm{~mm} / \mathrm{s}$ and matrix size of 128 by 64 . Finally, the repeatability error was about $1 \%$ for DT/ROI and $4 \%$ for DT/CROI at $50 \mathrm{~mm} / \mathrm{s}$ and acquisition size of 128 by 64 .

The reproducibility error was calculated as $0.54 \%$ for DT/ROI and $3.56 \%$ for DT/CROI. The lower enror for DT/ROI is explained, as the ROI does not include regions from the peripheral area of the flesh for the severely damaged fruits and thus the amount of analyzed tissue is reduced. These peripheral areas are characterized by the presence of higher magnetic susceptibility artifacts that explain the larger reproducibility errors for the DT/CROI ratios.

The ANOVA shows that for both ratios, DT/ROI and DT/CROI, a significant difference between undamaged and injured oranges at a $50 \mathrm{~mm} / \mathrm{s}$ belt speed exists (Table 4). No significant effect for repetition of test session (reproducibility) is found.

\section{Conclusions}

MRI in trials at a moving conveyor gave encouraging results for the on-line detection of freeze injury in oranges. Two belt speeds, 50 and $100 \mathrm{~mm} / \mathrm{s}$, as well as two acquisition data arrays, 128 by 64 and 128 by 32 (with acquisition times of 780 and $390 \mathrm{~ms}$, respectively) have allowed to discriminate between sound oranges and fruits severely affected by freezing conditions. The detection threshold has been bounded to $10 \%$ of damaged area compared to the entire tissue for static MRI and increased to $20 \%$ for images acquired under motion condition, $50 \mathrm{~mm} / \mathrm{s}$, and to $30 \%$ at $100 \mathrm{~mm} / \mathrm{s}$. The repeatability error varies from $1 \%$ to $5 \%$ for increasing belt speed. Reproducibility errors are bounded between 0.54 and $3.56 \%$. The ANOVA showed that the automated analysis of the images acquired in different days leads to similar ratios with significant differences between undamaged and damaged fruits. The optimization of this application involves the improvement of MRI under dynamic conditions to reduce motion artifacts in order to detect light freeze damages. 


\section{Acknowledgements}

We thank the Spanish Science and Technology Ministry for financing the project OPTIFRUT (AGL 2001-3792-C02-01) and Xavier Gimeno and Sandra Aguilar for destgning and building the conveyor.

\section{References}

1. Garcia-Ramos F.J., Ortiz-Cañavate J., Ruiz-Altisent M., Diez J., Flores L., Homer I., Chávez J.M.: J. Food Eng. 58, 53-57 (2003)

2. Kleynen O., Leemans V., Destain M.F.: Postharvest Biol. Technol, 30, 22l-232 (2003)

3. McCarthy M.J.: Magnetic Resonance Imaging in Foods, p. 3. New York: Chapman and Hall 1994.

4. Clark C.J., Hockings P.D., Joyce D.C., Mazzuco R.A.: Postharvest Biol. Technol. 11, I-21 (1997)

5. Zion B., McCarthy M.J., Chen P.: Lebensm.-Wiss. Technol. 27, 457-462 (1994)

6. Zion B., Chen P., McCarthy M.J.: COMPAG 13, 289-299 (1995)

7. Chen P., McCarthy M.J., Kin S.M., Zion B.: Trans. ASAE 39, 2205-2209 (1996)

8. Barteiro P., Moya A., Correa E., Ruiz-Altisent M., Fernández-Vaile M.E., Peirs A., Wright X.M., Hills B.P.: Appl. Magn. Reson. 22, 387-400 (2002)

9. Duce S.L., Carpenter T.A., Hall L.D.: J. Food Eng. 16, 165-172 (1992)

10. Gamble G.R.: J. Food Sci. 59, 571-573 (1994)

11. Ker W.L., Clark C.J., McCarthy M.J., de Ropp J.S.: Sci. Hortic. 69, 169-179 (1997)

12. Lufkin R.B., Bradley W.G., Brant-Zawadzaki M.: MR1: Principles and Artifacts, pp. 156-259. New York: Raven 1993. 